\title{
Editorial Review of Volume 20
}

Volume twenty of $\mathrm{CSSH}$ filled more pages than any of its nineteen predecessors; and that increased size, although not enough to make editorial choice any easier, allows somewhat greater variety within the rubrics used as categories of comparison. An editorial choice, those categories may have reinforced the impression that for the most part the essays published this year built on modes of comparison proved fruitful in the past: social institutions (law, family, church), the process of change (decolonization, modernization), and social roles (minorities, women). Most of these, particularly those dealing with social roles, are topics of dispute in contemporary affairs; and the articles published here often touched in scholarly and systematic terms on questions more angrily described in the public arena as racism, social control, or imperialism. In the long run the terminglogical temperature may not matter much, but there is a distinction worth making between comparison aimed at the resolution of precise questions formed from previous research and comparison used to identify the problems needing analysis, the recognition of which follows from some general interest or social concern. CSSH welcomes authors writing with the seriousness of engagement and willing to accept the risks of careful comparison.

This last issue of volume twenty adds another well-established category of comparison: social structure as reflected in (or an expression of) systems of land-holding and marketing. Given recurrent attention by economists, historians and anthropologists, this socio-economic nexus has gained new emphasis in the work of neo-Marxists and others particularly concerned with relations between the industrial and underdeveloped nations. Some examples of this emphasis are critically appraised in the review articles by Andrew Turton and Hilton Root. That the social effects of international markets can be surprising is neatly shown in Harriet Friedmann's application of formal theory to an apparent anomaly-in late nineteenth-century agriculture the specialized family farm won out over the large-scale use of wage labor. Her study adds to the growing recognition of how adaptable an economic unit the family can be (a point that emerged earlier this year in the articles in 20:3 by Gibbon and Curtin and by Minge-Kalman) and continues a discussion of the social effects of market relations to which Mintz (13:3) and Wallerstein (16:4) among others have made important contributions. Silvio Duncan Baretta and John Markoff bring a very current awareness of the instruments of social control to their treatment of the Latin American frontier, thus incidentally demonstrating how new 
insights emerge from the application of different (often current) concernsthe methodologically demanding would say from different models or paradigms - to comparative analysis. Katzman analysed the Brazilian frontier primarily in economic and ecological terms (17:3), and before that in the first volume of $\mathrm{CSSH}$ Gerhard (1:3) approached the study of frontiers in terms closer to those of Frederick Jackson Turner.

If there is a certain present-mindedness in the articles in this volume, they also exemplify the increasing interest among social scientists in using history and reassuringly show that the latest models of anthropologists and the latest methods of political scientists can also speak to those standing questions important to all students of society. As a whole, however, the articles in this issue do not break the tendency in much of current social science to so emphasize modern history as to risk exaggerating the differences between our world and pre-industrial society and to slight the independent study of culture (in fact, $\mathrm{CSSH}$ would especially welcome research attentive to earlier periods and to the complex relationships between high or formal and popular culture).

In this issue the subtle, sophisticated articles of Dale Eickelman and Daniel H. Levine do much to right the balance. Studies of institutions and at the same time attentive to values, they are also essays in the adaptation to social change of traditional cultures, a capacity emphasized by Shils in $13: 2$. More directly, Eickelman's essay adds to the analyses of education and of Islamic life as Levine's does to the analyses of Latin American politics and of Roman Catholicism, which have appeared in these pages.

Of course, every good piece of social analysis speaks to many aspects of society, and each article in CSSH may stimulate comparisons that sharpen by abrasion or illumine by contrast on topics quite different from those labelled by the rubrics in the table of contents. One of the functions of the editorial foreword to each issue is to point that out, to encourage continuing dialogues on many levels. The index that appears in this issue (the third; earlier ones were published in 15:4, for volumes $11-15$, and in $11: 4$, for volumes 1-10) should thus be used by readers for exploring other categories of comparison, and for tracing other themes of continuity. Much of the excitement of a scholarly journal lies in its community of discourse cutting across disciplines, areas, and time. Excitement is not what an index communicates best, but the reader who actually follows the trails it marks can more fully share in the many tiered dialogues, both public and private, that have been the life of CSSH in its first twenty years. 